\title{
Toward Personalized Cell Therapies by Using Stem Cells
}

\author{
Ken-ichi Isobe, ${ }^{1}$ Herman S. Cheung, ${ }^{2}$ and Ji $\mathrm{Wu}^{3,4}$ \\ ${ }^{1}$ Department of Immunology, Nagoya University Graduate School of Medicine, 65 Turumai-cho, Showa-ku, Nagoya, \\ Aichi 466-8550, Japan \\ ${ }^{2}$ Biomedical Engineering Department, College of Engineering, University of Miami, Coral Gables, FL 33146, USA \\ ${ }^{3}$ Key Laboratory for the Genetics of Developmental and Neuropsychiatric Disorders (Ministry of Education), \\ Bio-X Institutes, Shanghai Jiao Tong University, Shanghai 200240, China \\ ${ }^{4}$ School of Life Sciences and Biotechnology, Shanghai Jiao Tong University, Shanghai, China \\ Correspondence should be addressed to Ken-ichi Isobe, kisobe@med.nagoya-u.ac.jp
}

Received 16 November 2011; Accepted 16 November 2011

Copyright (๑) 2012 Ken-ichi Isobe et al. This is an open access article distributed under the Creative Commons Attribution License, which permits unrestricted use, distribution, and reproduction in any medium, provided the original work is properly cited.

Recent discovery of induced pluripotent stem cells (iPSCs) open the great possibility to use patient own tissue to the previously incurable diseases. Multipotent adult stem/progenitor cells have been identified in nearly all human organs. These cells are expanded in vitro and are possible to be used for personalized cell therapy. This special issue collected the articles which discussed recent progresses in this field.

Our body has self-defense systems to recover from damages. However, once tissues are damaged extensively, damaged tissues cannot be recovered. These include hepatic (C. H. Chiang, M. A. Puglisi), kidney, lung, and heart failures. Also in neurodegenerative diseases including Parkinson's disease, Alzheimer diseases, and ALS, patient cells are difficult to recover (H. Y. Ha). External stimuli such as high dose of irradiation caused by cancer treatment (B. Phulpin) will induce irreversible damages. There exist genetic disorders for which there is no cure including progressive muscular dystrophies (E. Zucconi), cystic fibrosis (V. Paracchini). Further age-related dysfunctions are difficult to be recovered. One example is articular cartilage (S. Seo). Currently transplantation has been shown to be an effective treatment for these tissue failures. However, transplantation has serious problems: shortage of organs to be transplanted and immune rejection caused by great heterogeneity of human HLA. Recent progresses of biotechnology open a new hope to treat incurable diseases by using patient own tissues. This special issue aimed to gather scientific papers about recent progresses in this field.

iPSCs are novel stem cell populations induced from mouse and human adult somatic cells through reprogramming by transduction of defined transcription factors. These cells open the great possibility to personalized cell therapy to treat incurable diseases (K. Seiler, T. Kunkanjanawan, H. Y. Ha). Compared to ES cells, iPSCs are not rejected by host immune cells theoretically. At least iPS cells are not rejected once these cells are differentiated (C. H. Chiang).

Mesenchymal stromal cells (MSCs) from various sources including bone marrow (S. W. Gendebien), adipose tissue (W. Lattanzi), dental pulp, placenta, umbilical cord (E. Zucconi, S. Park), menstrual blood (M. C. Rodrigues) and amniotic mesenchymal stromal cells (V. Paracchini) have been studied extensively by animal models.

Tissue stem cells are also used for personalized stem cell therapy. Muscle-derived stem cells for bone formation (X. Li), blood vessel-derived stem cells regenerate myofibers in injured and dystrophic skeletal muscles as well as improve cardiac function after myocardial infarction (C. W. Chen). Olfactory ensheathing cells (OECs) into nerve or spinal cord injuries can promote axonal regeneration and remyelination and restore functional recovery (C. Radtke). Some stemlike cells reside in the transition area between the peripheral corneal endothelium (CE) and the anterior nonfiltering portion of the trabecular meshwork (TM) replacing the lost CE (W. Y. Yu).

Cell culture on scaffold enhances cell growth and differentiation. Hydroxyapatite (HA) coating of (PCL/PVA) nanofibers enhances bone repair (E. Prosecka).

There are a number of reports for limitations of current technologies that hinder iPSCs into practical use. Immunogenicity to syngeneic mice and tumor formation are reported. MSCs also might promote tumor growth. MSCs 
immunomodulatory properties affect greatly the host. More works are sure to be needed to overcome these problems.

\section{Acknowledgments}

We thank all of the authors for submitting their papers to this special issue and appreciate their efforts and time in processing their papers. We also thank all the reviewers for careful checking of the submitted papers.

Ken-ichi Isobe

Herman S. Cheung

$\mathrm{JiWu}$ 

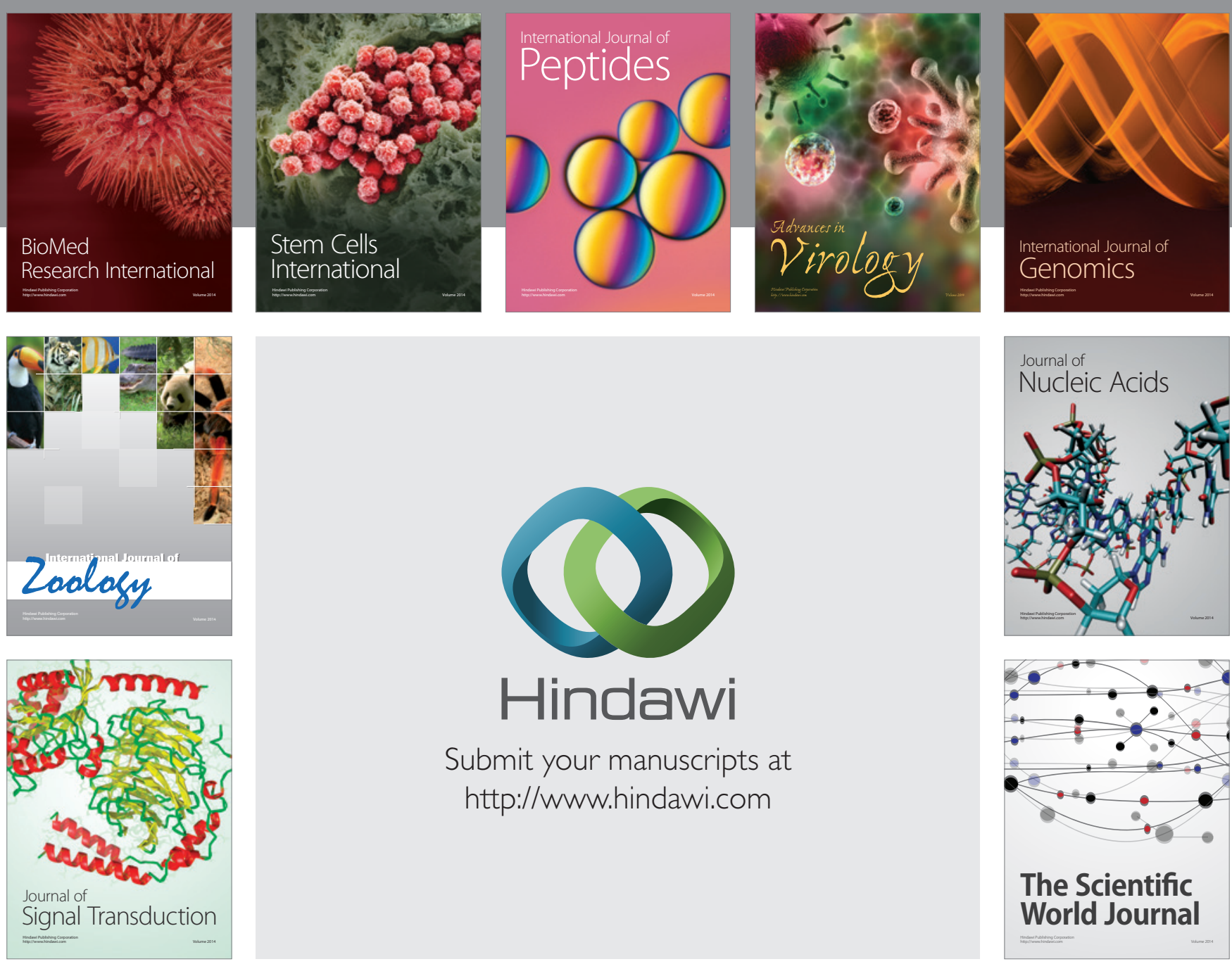

Submit your manuscripts at

http://www.hindawi.com
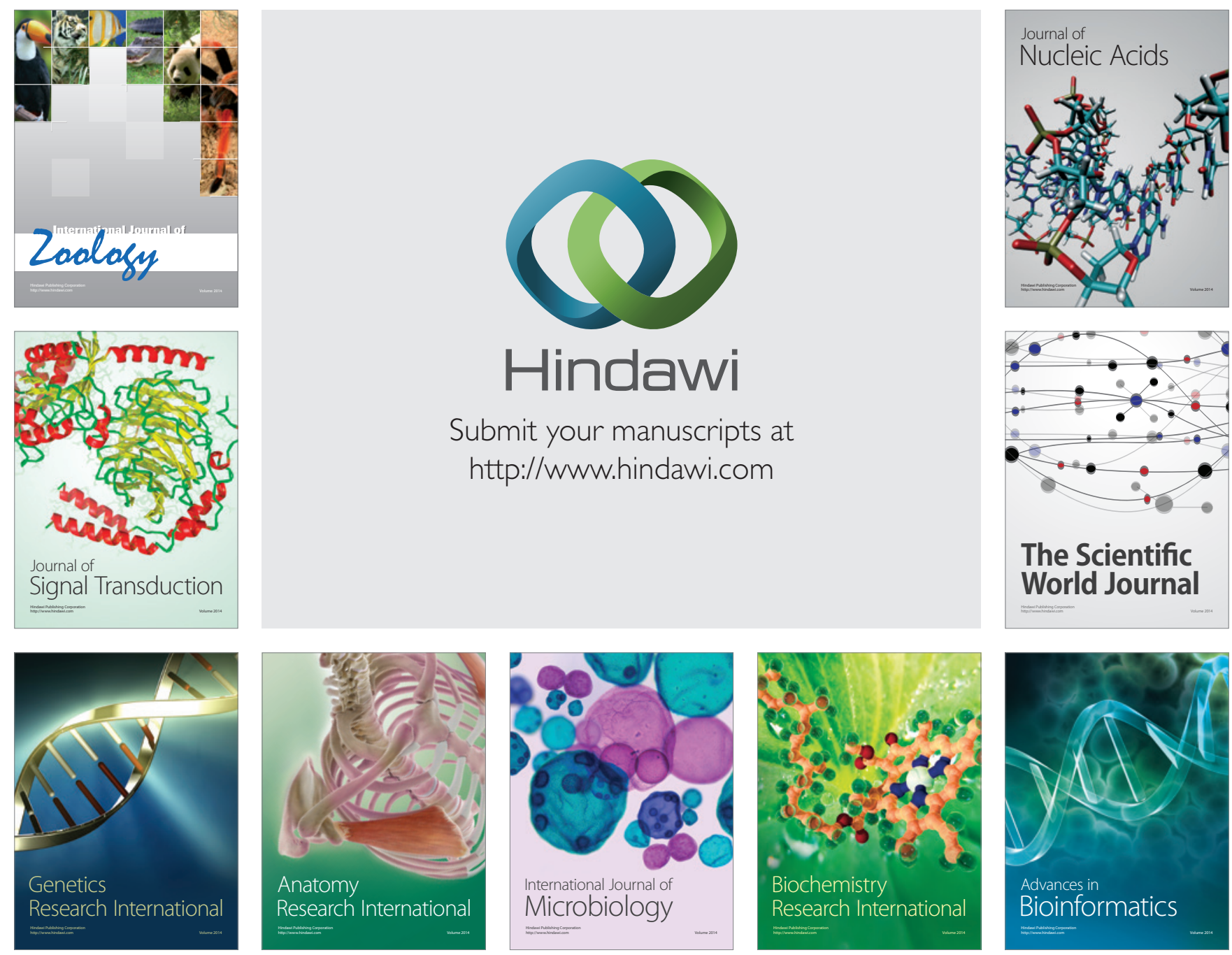

The Scientific World Journal
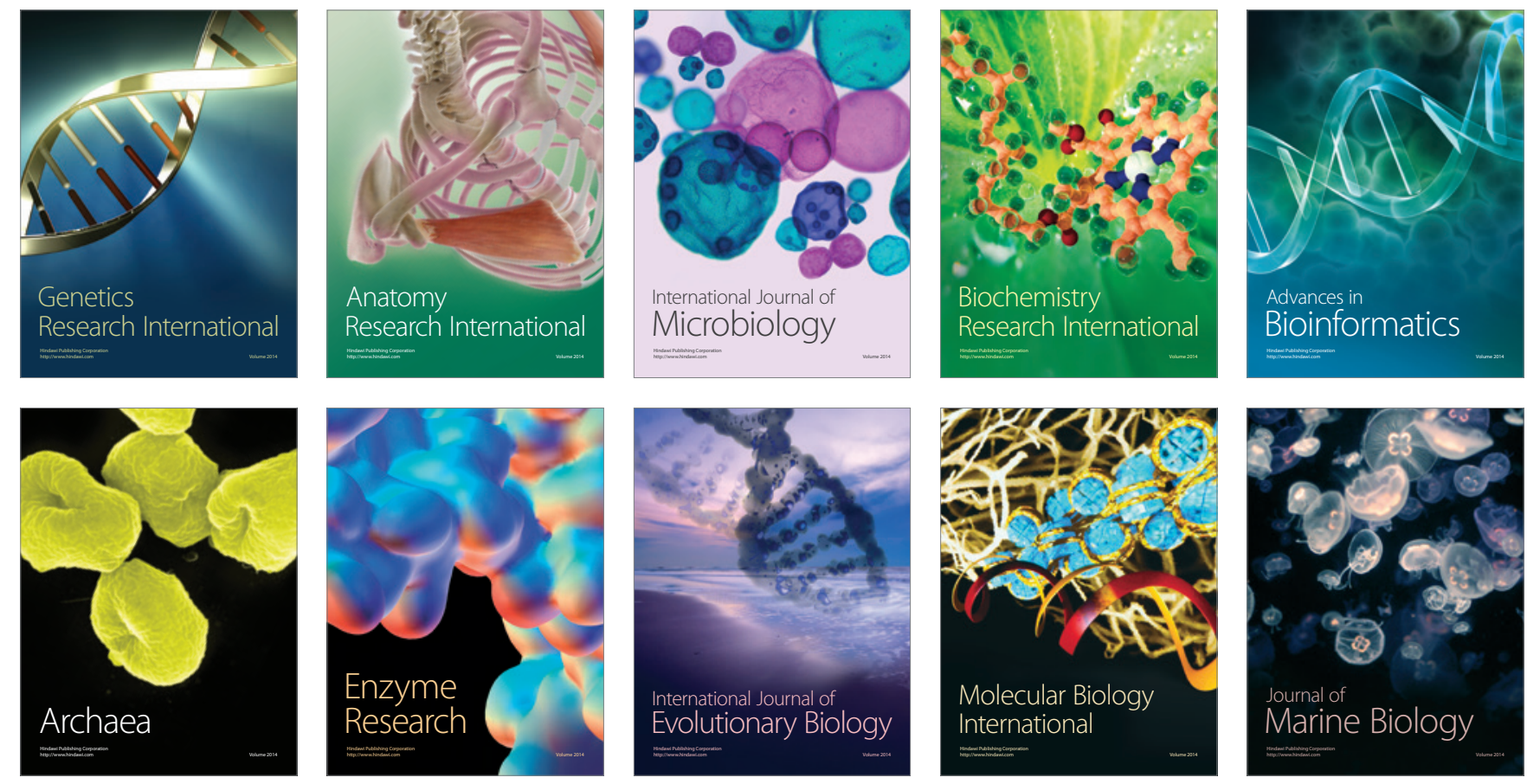